Revue internationale P.M.E.

Économie et gestion de la petite et moyenne entreprise

\title{
La revitalisation des régions de tradition industrielle : le rôle des PME et du milieu
}

\section{Denis Maillat}

Volume 1, numéro 1, 1988

URI : https://id.erudit.org/iderudit/1007871ar

DOI : https://doi.org/10.7202/1007871ar

Aller au sommaire du numéro

Éditeur(s)

Presses de l’Université du Québec

ISSN

0776-5436 (imprimé)

1918-9699 (numérique)

Découvrir la revue

Citer cet article

Maillat, D. (1988). La revitalisation des régions de tradition industrielle : le rôle des PME et du milieu. Revue internationale P.M.E., 1(1), 7-22.

https://doi.org/10.7202/1007871ar

\section{Résumé de l'article}

Certaines régions de tradition industrielle ont montré qu'elles pouvaient revitaliser leur système de production. Elles sont capables de mutations (la mutation est un événement à caractère plus ou moins aléatoire qui introduit un changement à l'intérieur des éléments constitutifs d'une entité). Ces mutations se manifestent par une recombinaison de leurs composantes qui, en liaison avec une partie du potentiel d'origine, donne naissance à un caractère nouveau. Il est donc important d'analyser les capacités d'un tissu industriel traditionnel à provoquer des phénomènes d'adaptation et d'innovation technologique. A partir d'une impulsion initiale, on observera comment les entreprises adaptent leur processus de production, en tenant compte des compétences, des opportunités et du potentiel existant dans leur environnement. Cette recomposition du système économique local, dépend de l'émergence des PME, de leur ancrage régional et du milieu. 


\title{
La revitalisation des régions de tradition industrielle : le rôle des PME et du milieu
}

\author{
Denis MAILLAT \\ Directeur, Institut de Recherches Economiques et Régionales \\ Université de Neuchâtel
}

\begin{abstract}
RESUME
Certaines régions de tradition industrielle ont montré qu'elles pouvaient revitaliser leur système de production. Elles sont capables de mutations (la mutation est un événement à caractère plus ou moins aléatoire qui introduit un changement à l'intérieur des éléments constitutifs d'une entité). Ces mutations se manifestent par une recombinaison de leurs composantes qui, en liaison avec une partie du potentiel d'origine, donne naissance à un caractère nouveau. Il est donc important d'analyser les capacités d'un tissu industriel traditionnel à provoquer des phénomènes d'adaptation et d'innovation technologique. A partir d'une impulsion initiale, on observera comment les entreprises adaptent leur processus de production, en tenant compte des compétences, des opportunités et du potentiel existant dans leur environnement. Cette recomposition du système économique local, dépend de l'émergence des PME, de leur ancrage régional et du milieu.
\end{abstract}

\begin{abstract}
Some traditional areas have proved themselves to be capable of revival. They are capable of undergoing mutations (a mutation being a somewhat random event that produces a change within the elements of a given entity). These mutations manifest themselves through the reorganization of an area's component which, combined with certain aspects of its original potential, produces a whole new character it is therefore important to analyse the exent to which the traditional industrial fabric is capable of provoking technological adaptation and innovation. Based on the initial impetus, it is possible to observe how companies adapt their production process by taking into account their abilities, their opportunities and their environmental potential. This reorganization of the local economic system is dependent upon the emergence of SME's and their foothold in a given region and on the environment itself.
\end{abstract}

\section{RESUMEN}

Ciertas regiones de produccion industrial han demostrado poder revivificar su sistema de produccion. Ellas son capaces de mutaciones (siendo la mutacion un evento de caracter mas o menos aleatorio que introduce un cambio al interior de los elementos constituyentes de una entidad). Estas mutaciones se manifestan por medio de una reorganizacion de sus componentes, que combinada con una parte del potencial original produce un caracter nuevo. Es por eso importante analyzar la capacidad de un tejido industrial tradicional para provocar fenomenos de adaptacion y de innovacion tecnologicas. A partir de un impulso inicial se puede observar como las empresas adaptan su proceso de produccion al tener en cuenta la competencia, las oportunidades y el potencial existente en su medio ambiente. Esta recomposicion del sistema economico local depende de la emergencia de las P.M.E. de su sujecion regional y del medio. 
Depuis une dizaine d'années, la géographieéconomique des pays développés a subi un certain nombre de modifications. De nouvelles régions dynamiques apparaissent alors que des régions jusque-là riches s'appauvrissent.

Ce renversement des hiérarchies spatiales traditionnelles se manifeste dans plusieurs pays industrialisés. Aux États-Unis, le mouvement va de la côte est vers l'ouest et le sud. En Italie, les trois premières provinces qui occupaient un quart de l'emploi industriel en 1971 n'en ont plus qu'un cinquième en 1981. En Suisse, où des régions à tradition touristique comme le Tessin ou rurale comme Fribourg, ont tendance à devenir attractives au détriment des régions plus industrielles. En France également où les onze régions les mieux placées de 1968 à 1975 ont, au cours de la période 1975 à 1981, des performances en matière d'emplois moins bonnes que les 10 dernières régions. L'orientation de l'activité vers les régions considérées comme les moins riches (PACA, Languedoc-Roussillon, Centre) au détriment de celles de vieille tradition industrielle (Lorraine, Nord - Pas-de-Calais, Ile-de-France) est manifeste (Aydalot, 1986).

Pour les régions de tradition industrielle, la situation paraît donc difficile. Elles perdent de l'importance par rapport à des régions nouvelles, à de nouveaux foyers d'innovation. Toutefois, bien que l'époque actuelle porte en elle le mécanisme du retournement des hiérarchies spatiales, cela ne veut pas dire que tous les espaces industriels vont devenir pauvres et dominés. En effet, certaines régions de tradition industrielle sont capables de revitaliser leur système de production. Cette recomposition du système économique local, dépend de l'émergence des PME, de leur ancrage régional et du milieu.

\section{PME et milieu}

\subsection{L'émergence des PME}

La création de PME est considérée aujourd'hui comme indispensable à la revitalisation et au développement des tissus économiques régionaux. Depuis le début des années 1970, il apparaît que la plupart des nouvelles entreprises sont de petites entreprises (Keeble et Wever, 1986), aussi bien dans les branches industrielles que dans celles des services. En outre, ces nouvelles entreprises ont généralement été créées par des entrepreneurs locaux (Storey, 1986, Maillat, 1986). Dans le même temps, on relève des carences chez les grandes entreprises, tant en ce qui concerne la création d'emplois que d'établissements nouveaux. On estime en outre que, dans les grandes entreprises, l'esprit entrepreneurial et les projets d'innovation sont freinés par les complications administratives et par le poids des structures. Ceci expliquerait qu'elles réagissent souvent lentement aux modifications de l'environnement. En revanche, dans le contexte actuel d'émergence de technologies nouvelles et d'incertitude sur les marchés, les PME sont considérées comme des 
agents privilégiés du changement technologique et de l'innovation. Ainsi, en raison de la complexité structurelle et de la pesanteur décisionnelle des grands ensembles, les PME sont, à bien des égards, plus aptes à s'adapter aux avances technologiques ou aux variations de la demande, d'autant plus que la robotisation a rendu possible la fabrication de petites séries à des coûts raisonnables.

\subsection{L'ancrage régional des PME}

Traditionnellement, on avait pris l'habitude d'attribuerà la grande entreprise un rôle primordial dans le développement régional par les effets d'entraînement qu'elle était susceptible de provoquer. On procédait volontiers à une analyse dualiste des activités économiques en opposant un secteur composé de grandes entreprises innovantes à un secteur réunissant les PME considérées comme des sous-traitants et de faible niveau technologique (Pecqueur, 1986).

Or, aujourd'hui, certaines PME sont particulièrement dynamiques et font preuve d'un développement autonome. Leur émergence n'est pas due à leur appartenance à un réseau de sous-traitance dominé par les grandes entreprises. Elles ont des caractéristiques qui en font des agents actifs de l'innovation technologique et de la reconstitution des systèmes économiques. Certes, il ne s'agit pas d'opposer systématiquement les PME aux grandes entreprises. Ces dernières restent des facteurs déterminants dans l'évolution des systèmes de production. Toutefois, du point de vue d'une région, le rôle des nouvelles PME est stratégique. Elles lui permettent d'échapper à la logique de la division fonctionnelle du travail ou d'en diminuer l'impact. La région dispose ainsi d'éléments déterminants pour reconstituer son système de production puisque les PME ont une assise locale et un ancrage territorial.

\subsection{Le rôle du millieu}

Les PME ne fonctionnent pas isolément les unes des autres : elles appartiennent à un réseau qui, pour être efficace, doit avoir une dimension territoriale (Planque etPy, 1986, Maillat, 1986). On peut donc faire l'hypothèse du «rôle déterminant joué par les milieux locaux comme incubateurs de l'innovation, comme prisme à travers lequel passeront les incitations à l'innovation et qui donne sur le terrain son visage à celle-ci; l'entreprise n'est pas un agent innovateur isolé; elle est partie du milieu qui la fait agir. Le passé des territoires, leur organisation, leur comportement collectif, le consensus qui les structure sont des composantes majeures de l'innovation» (Aydalot, 1986). Ainsi, les PME sont engagées avec d'autre partenaires dont les collectivités locales, dans un jeu social local dans lequel les positions de chacun ne peuvent être expliquées seulement par la nature des processus de production ou par le type de produit et de marché (Raveyre et Saglio, 1984). Le système industriel local est donc davantage que la simple juxtaposition d'unités de production; c'est un ensemble de liens entre les instances politiques et économiques. Par le biais de réseaux multiples de communication, les territoires produisent une solidarité entre 
les acteurs locaux.Celle-cis'exprime en particulier dans les stratégies entrepreneuriales de diffusion de l'innovation (Pecqueur, 1986).

Dès lors, la réussite des PME est déterminée par les caractéristiques et le dynamisme du milieu dans lequel elles sont implantées. Le territoire d'implantation devient pour elles un espace de solidarité capable de générer des compétences et des ressources territoriales spécifiques. Ces spécificités territoriales jouent un rôle beaucoup plus important pour les PME que pour les grandes entreprises généralement moins concernées par l'environnement du lieu d'implantation de leursétablissements. Ainsiapparaîtl'importance du milieu, du territoire, de l'environnement du producteur, dans la revitalisation des régions industrielles et dans la réorganisation de leur développement. Tenir compte de la variable territoriale, c'est renouveler les conceptions traditionnnelles du développement régional.La valorisation des potentiels locaux aboutità un modèle de développement différent qui peut relayer la défaillance du modèle de croissance par la grande entreprise (Pecqueur, 1986). En effet, si les grandes entreprises contrôlent l'innovation, la localisation de l'activité nouvelle sera d'emblée intégrée à une logique de division spatiale du travail, alors que si des initiatives multiples prennent le dessus, c'est une forme originale de développement qui apparaît (Aydalot, 1986). La dynamique du développement territorial est le résultat de la capacité d'entreprendre locale et non la conséquence de la mobilité interrégionale des entreprises.

Il ne faut toutefois pas en déduire que seule la dimension territoriale du développement doit être prise en compte. En effet, une région est ouverte, elle ne vit pas en vase clos. Son système de production fonctionne par conséquent selon une double logique : une logique horizontale ou territoriale et une logique verticale ou fonctionnelle (Perrin, 1986). Il ne s'agit pas d'opposer ces deux logiques mais de trouver les articulations qui s'établissent entre elles. C'est d'ailleurs un des problèmes fondamentaux posés à la politique régionale. Comme l'écrit Pecqueur (1986), «la tentation est grande de substituer à l'approche fonctionnelle une vision exclusivement horizontale. Toutefois, la variable territoriale ne peut pas être le seul élément structurant de l'espace économique. On ne peut en effet profiter des potentiels territorialisés qu'en confrontant les territoires aux logiques fonctionnelles et en cherchant non pas le repli du local sur lui-même mais une combinaison de synergies pour l'adaptation innovante du local à ces logiques contraignantes».

On peut donc envisager l'innovation :

- dans une optique fonctionnelle. Elle est alors un produit ou un procédé nouveau, défini par des paramètres techniques et ayant vocation d'être appliquée partout de façon standardisée.

- dans une optique territoriale. L'innovation passe par la création d'un milieu capable de répondre à un défi par l'utilisation de l'expérience locale. Elle est le fruit de l'inventivité du milieu (Aydalot, 1986).

Dans cette dernière perspective, le plus important n'est pas l'apparition d'une nouvelle technique mais la décision de donner vie au processus territorial d'innovation. Celui-ci ne dépend pas de la présence dans la région d'agents ou de 
ressources particuliers mais de leur interaction mutuelle. L'innovation et le processus de production qui la génèrent doivent être vus comme l'expression changeante du milieu. Le milieu dans lequel est implantée l'entreprise s'intègre au fait productif. Il n'est plus une donnée, une contrainte, mais une variable stratégique dans le processus d'innovation (Gaffard, 1986). Dès lors les milieux apparaissent comme les incubateurs des innovations et des entreprises innovantes. Les comportements innovateurs ne sont pas seulement nationaux, ils dépendent aussi de variables définies au niveau local et régional (Aydalot, 1986). On peut de ce fait considérer chaque milieu comme capable de générer un processus d'innovation spécifique.

Plutôt que d'aborder l'innovation régionale par les milieux, on pourrait partir de l'entreprise ou des technologies. L'approche par l'entreprise implique que l'on s'intéresse aux facteurs qui déterminent sa localisation. On peut ainsi montrer que lesentreprises nouvelles (notammentcelles qui incorporent delahaute technologie) ont des comportements de localisation différents des activités plus anciennes. On arrive rapidement à la conclusion que les conditions de localisation offertes par les régions industrielles traditionnelles ne permettent pas une implantation spontanée de ces nouvelles entreprises. Une politique d'attraction est toujours possible, mais elle contribue à maintenir la dynamique technologique à l'extérieur de la région (Perrin, 1986a, Maillat et al., 1984). L'approche par les technologies (celles-ci étant considérées comme exogènes) amène à s'interroger sur l'aptitude des régions à les capter. Elle ne permet pas de mettre en évidence les processus d'innovation. En définitive, seule l'approche par les milieux porte l'accent sur le processus territorial d'innovation et la capacité de chaque milieu à secréter la forme de progrès technique adapté à son passé et à ses structures.

Ceci permet de mieux comprendre pourquoi l'innovation n'est pas réservée aux seules régions où prédominent les hautes technologies. D'autres régions sont capables d'innovation, même si elles ont un passé industriel (Stöhr, 1986). On ne peut en effet «se borner à affirmer, écrit Aydalot (1986), que les tissus industriels constitués de longue date sont incapables d'innover». Le défi d'une technologie d'origine extérieure, le risque de ruine entraînent des réactions dont certaines sont des réponses satisfaisantes et orientées vers l'innovation.

Les études du GREMI ont bien montré que des régions industrielles comme celles de Saint-Étienne, (Peyrache, 1986) de l'Arc jurasssien, (Maillat et Vasserot, 1986) de Franche-comté, peuvent trouver les ressources nécessaires à leur revitalisation. Ces tissus industriels traditionnels innovent par filiation-continuité et ne bouleversent jamais tout à la fois le marché, le produit ou la technologie. Lorsqu'existe un tissu industriel à base d'entreprises petites et moyennes, occupant des travailleurs d'un niveau élevé de qualification, lorsque certaines continuités technologiques sont possibles, les vieux milieux industriels peuvent survivre à une «agression technologique» majeure. Mais le nouvel équilibre vers lequel ils tendent sera obtenu à un niveau d'activité moindre (notamment lorsque celui-ci est mesuré en termes d'emplois). Par ailleurs, le fait que ces milieux soient caractérisés par une forte organisation collective a facilité leur reconversion. Ainsi, le consensus local et 
la prise en main collective de l'avenir sont déterminants. En somme, dans les milieux à tradition industrielle, l'innovation ne peut réussir que si elle repose sur des éléments de continuité avec l'expérience acquise par le milieu. Paradoxalement, l'innovation technologique qui signifie d'abord rupture avec le passé doit extraire de ce passé des éléments de continuité qui faciliteront son insertion, réduiront les résistances et augmenteront ses chances de diffusion (Aydalot, 1986).

Dans ces régions, les systèmes territoriaux recèlent des ressources et des compétences potentielles et économiquement actualisables. Pour cela, elles doivent s'insérer dans de nouveaux espaces fonctionnels de production tout en transformant l'aspect territorial de leur système de production afin de lui permettre, non pas d'être un simple réceptacle de nouvelles technologies, mais de devenir coproducteur de la technologie et de l'innovation (Perrin, 1984, Maillat 1986).

\section{Le processus de revitalisation des régions de tradition industrielle}

Leprocessus de revitalisation des régions de tradition industrielle caractérisées par de nombreuses PME et un savoir-faire spécifique, implique que trois sousensembles interreliés de leur milieu subsistent :

- L'appareil territorial de production (nature des entreprises et de leur insertion régionale, cohérence de l'ensemble des activités, etc...),

- Les chaînes de mobilité du marché du travail,

- L'appareil scientifique régional (systèmes de formation, instituts de recherche...).

L'hypothèse est qu'il existe des liens entre les trois groupes d'éléments précités, que ces liens constituent des réseaux qui véhiculent, diffusent et renouvellent les compétences et les impulsions indispensables à la revitalisation.

\subsection{L'apparell territorialisé de production}

\subsubsection{Le changement technologique et l'innovation}

Pour comprendre le processus de revitalisation des régions de tradition industrielle, il ne faut pas être obnubilé par la haute technologie. Il s'agit en effet de faire la différence entre la fonction d'usage d'une technologie et la fonction de production de technologie (Quévit, 1985). Les régions productrices de technologies nouvelles sont assez peu nombreuses; ce n'est en revanche pas le cas pour celles qui sont susceptibles d'utiliser de nouvelles technologies. Dans le même esprit, il faut distinguer l'innovation qui consiste en l'amélioration de produits et de procédés «incremental innovation» de l'innovation radicale qui amène un changement révolutionnaire. 
Dans les régions industrielles, on rencontre surtout le premier type d'innovation. Par exemple, dans l' Arc jurassien, trois-quarts des innovations effectuées dans les produits relèvent d'une amélioration d'un produit existant, tandis que le quart restant se répartit pour moitié entre innovations par diversification et innovations par bifurcation dans des produits de pointe (Maillat et Vasserot, 1986).

Ces innovations sont, certes moins spectaculaires que des innovations radicales, mais elles sont déterminantes pour l'avenir des entreprises concernées. Elles amènent une différenciation du produit qui permet le maintien ou l'accroissement des parts de marché. Elles sont la preuve que les entreprises sont capables d'assimiler et d'utiliser les nouvelles technologies. Ce n'est donc pas seulement les innovations majeures ou fondamentales qui sont importantes pour le maintien de l'avance technique d'une région, mais tout autant la mise-à-jour et l'adaptation technique des produits fabriqués dans la région, tant il est vrai que les industries traditionnelles sont des industries d'avenir dès lors qu'elles renouvellent leurs produits et leur procédés de production.

\subsubsection{L'entreprise innovatrice et son ancrage régional}

La distribution régionale des innovations de produits ou de procédés dépend dans une large mesure des caractéristiques des entreprises et plus particulièrement de leur capacité à procéder à des changements technologiques (Boulianne et Maillat, 1983). De ce fait, du point de vue du processus territorial de revitalisation, les entreprises capables d'innover sont plus importantes que les autres. On ne peut pas, en effet, comparer une entreprise qui fabrique des produits sophistiqués à une discothèque ou à un garage. Parmi la population très hétérogène des PME, il faut faire une sélection et identifier les entreprises innovatrices.

Le processus territorial d'innovation est largement en rapport avec ce type d'entreprises bien qu'elles représentent rarement plus de $10 \%$ du total des entreprises. Il est évident qu'il est important que ces entreprises aient leur centre de décision dans la région. On sait en effet que l'ancrage régional et les relations avec les autres entreprises sont moins grands pour les établissements appartenant à un groupe que dans le cas d'une entreprise mono-établissement. On peut, par ailleurs, établir le profil des entreprises innovatrices (du moins dans les régions industrielles que nous avons étudiées) en retenant les caractéristiques suivantes (Maillat, 1986).

\subsubsection{La personnalité de l'entrepreneur}

La vitalité d'une PME dépend beaucoup de la personnalité de l'entrepreneur. Sans entrer dans les détails, une de ces qualités réside dans son savoir-faire économique (découverte d'opportunités, développement de stratégies, identification des ressources et des compétences). Ces savoir-faire s'acquièrent le plus souvent par l'activité professionnelle. C'est ainsi que la plupart des créateurs d'entreprises ont une expérience professionnelle antérieure (Keeble et Wever, 1986, Maillat, 1986). 
Cette caractéristique est particulièrement évidente lorsqu'il s'agit de «technical entrepreneurship».

L'émergence d'entrepreneurs dépendégalement du milieu.En effet, beaucoup d'entrepreneurs commencent leur activité par rapport à quelque chose qui leur est familier. De ce fait, une relation existe entre les nouvelles PME et les entreprises existantes. En outre, dans les régions où figurent beaucoup de $\mathrm{PME}$, la probabilité de voir apparaître des créateurs d'entreprises semble plus grande que dans les régions dominées par les grandes entreprises.

\subsubsection{L'organisation interne des entreprises innovatrices}

Si la personnalité de l'entreprise est importante, la manière dont est organisée la PME l'est tout autant. On sait, en effet, que la capacité d'innovation dépend largement des dépenses effectuées pour la recherche et le développement, la mise au point de prototypes, l'ingénierie, le contrôle de la qualité, la vente, le marketing. C'est avec ce type de personnel que l'entreprise constitue son capital humain et donc le savoirfaire spécifique de sa «matière grise» (Maillat, 1986, Decoster et Tabariès, 1986).

\subsubsection{L'organisation du changement et l'utilisation des services extérieurs "professional services»}

Les PME innovatrices sont caractérisées par leur volonté de faire face au changement technologique et de le gérer. Les plus dynamiques sont celles dont la politique de développement ne dépend pas d'une seule personne (comme c'est souvent le cas dans les entreprises de type familial) et qui intègrent des sources extérieures de connaissances et de know-how à leur développement technologique (Boulianne et Maillat, 1983). Il y a donc un lien entre le degré d'innovation et l'appel à l'extérieur (Meyer - Krahmer, 1985). Ce sont les entreprises technologiquement les plus avancées qui ont le degré d'ouverture le plus grand. Elles cherchent leur information (laboratoires de recherche, consultants, experts, etc) et la trouvent quelle que soit sa source (c'est-à-dire à proximité immédiate ou dans une région plus éloignée). Ce qui caractérise ces entreprises c'est le contact formel et quasi continu avec les sources d'information. Meyer-Krahmer, (1985) distingue par ailleurs un groupe d'entrepreneurs au degré d'ouverture moyen. Il s'agit d'entreprises qui, bien qu'elles innovent, n'ont que des contacts ponctuels, informels, avec les fournisseurs d'information. On constate cependant que la présence, au niveau local, de fournisseurs de know-how, d'universités, d'écoles techniques, etc... conduit ces firmes à mieux exploiter des possibilités.

\subsubsection{Les PME et l'exportation}

On a souvent considéré que les PME étaient des entreprises sous-traitantes et qu'elles exportaient peu. Aujourd'hui, pour nombre d'entre elles, ce n'est plus le cas. La PME innovatrice est une entreprise ouverte au monde et qui exporte. Elle est 
spécialisée et il n'est pas rare qu'elle détienne un monopole de sa spécialité. Autrement dit, une des principales différences entre les firmes innovatrices et celles qui n'innovent pas est la dimension spatiale et l'internationalisation de leur marché (Meyer-Krahmer, 1985, Maillat, 1986, Decoster et Tabariès 1986).

\subsubsection{L'existence de filière(s) et de services aux entreprises}

Dans une région de tradition industrielle, les entreprises ne sont pas des acteurs isolés; elles sont en général reliées les unes aux autres dans le cadre de filières ou de parties de filières. Ce sont ces liens qui déterminent la cohérence des systèmes productifs régionaux, notamment dans les régions où il existe beaucoup de PME. Cette cohérence résulte des principes d'organisation qui caractérisent les filières. Elle donne un sens aux effets de complémentarité et d'interdépendance techniques et économiques. Ainsi, dans les régions de tradition industrielle que nous analysons ici, les capacités de changement ne résultent pas de la spécialisation sur un créneau particulier, mais d'une trame d'activités interdépendantes qui suscite la sous-traitance et le partenariat. De ce point de vue, le concept de filière indique que «en regardant ce qui permet la production d'un produit fini (une montre, une voiture), on ne peut étudier uniquement l'industrie horlogère ou l'industrie automobile mais on doit analyser tout «l'arbre» de l'activité à travers les secteurs et les branches» (Lamboy, 1984).

Par ailleurs, il faut tenir compte du fait que les dynamiques territoriales sont couplées à des dynamiques fonctionnelles nationales et internationales. Cette intégration est assurée par l'intermédiaire des filières ou des parties de filières présentes dans une région.

On l'a vu dans le cas de la région horlogère qui a pu se raccrocher à la filière électronique (Maillat, 1984). Des exemples semblables existent dans les régions où dominent la marchine-outil ou le textile. Par rapport au processus territorial d'innovation, la possibilité de l'incorporation d'une nouvelle partie de filière (électronique par ex.) dans une filière plus traditionnelle (mécanique par ex.) est essentielle. Certes, l'impact d'une telle évolution est souvent négatif en terme d'emplois; toutefois il est positif en terme de possibilités de revitalisation puisqu'une nouvelle technologie est ainsi introduite dans le sytème productif régional. A partir de là de nouvelles interdépendances peuvent se développer et de nouveaux effets d'entraînement apparaissent ou se renouvellent sans que la cohérence traditionnelle du système de production soit totalement éliminée. Dans ce sens, il y a une filiation entre le système productif régional traditionnel et celui qui est en train de se dessiner. Cette filiation se manifeste notamment par l'utilisation des savoir-faire traditionnels qui sont renouvelés au contact des savoir-faire nouveaux. Grâce à leur appartenance à la filière, les entreprises nouvelles et anciennes peuvent profiter de ces compétences régionales.

La mise en évidence de l'interdépendance entre activités a conduit peu à peu à dépasser la notion purement industrielle de la filière. En effet, les systèmes productifs se développent toujours plus dans le sens de l'intégration entre activités 
de fabrication et activités de services, notamment de services aux entreprises (Bailly et Maillat, 1986). Aux différents niveaux des filières de production, il est nécessaire d'ajouter de nouvelles fonctions de services, tant en amont qu'en aval de la fonction de fabrication (recherche, management, marketing) (Bailly et Maillat, 1986, Lamboy et Tordoir, 1985, Preel, 1986). Cette évolution reflète la nécessité d'établir un lien entre l'information et l'aspect physique de la production : «l'information est une structure composée d'éléments physiques dans un système de production, combinant, coordonnant et contrôlant leurs relations. Elle joue un rôle intégrateur reliant par exemple les actions de la firme dans son environnement avec ses opérations (physiques) internes. C'est pourquoi les services professionnels créent un interface entre le corpus physique interne d'une firme - sa sphère de production - et le monde extérieur» (Lamboy et Tordoir, 1985).

Le développement de ces compétences et de ce savoir-faire tertiaire ne devrait pas échapper aux régions industrielles sinon leur sytème de production resterait incomplet et les priverait d'éléments indispensables à la dynamique du processus d'innovation. Ceci pose évidemment le problème de la localisation de ces activités de services aux entreprises. Souvent, on a relevé que les activités de services évoluent dans une structure spatiale différente des activités de type secondaire. Si pendant longtemps, la localisation des services aux entreprises a été principalement concentrée dans les agglomérations, ce n'est plus toujours le cas aujourd'hui. Ces services peuvent se développer à d'autres niveaux de la hiérarchie urbaine (Bailly, Maillat et Rey, 1984). Cette évolution est d'autant plus significative que les zones en question comprennent beaucoup de PME. En effet, celles-cine peuvent pas développer à l'intérieur toutes les fonctions de service dont elles ont besoin, aussi font-elles appel à l'extérieur. La demande de services aux entreprises est donc croissante. Cette augmentation sera d'autant plus grande que pour assurer leur pérennité les PME doivent constamment renouveler leurs produits et demeurer dynamiques. En conséquence, la dimension des aires de marché pour rentabiliser un service spécialisé devient de moins en moins grande. Cette évolution devrait donc favoriser les localisations de proximité capables de compléter les filières traditionnelles (Lamboy et Tordoir, 1985).

\subsection{Les chaînes de mobilité du marché du travall}

Le marché du travail est un élément essentiel du potentiel d'une région industrielle puisque, à travers la main-d'oeuvre et ses qualifications, il représente un certain volume de capital humain, c'est-à-dire un (ou des) savoir-faire spécifique(s). Par ailleurs, les différents types d'emplois déterminent l'insertion des individus dans leur région.

Les différentes approches du développement territorial portent leur attention surle capital local humain, c'est-à-dire sur les talents, les initiativeset lesconnaissances des habitants de la région. Elles considèrent que ces ressources locales sont capables de donner ou de développer un avantage comparatif pour la région (Coffey et Polèse, 
1984). Mais le capital humain est mobile : il peut se déplacer d'une région à l'autre. Dès lors, pour une région, c'est sa capacité à retenir son capital humain qui est déterminante. Cela n'est possible que si le marché du travail offre des opportunités suffisantes.

La taille, la structure, les caractéristiques du marché du travail (qualifications demandées, possibilités d'avancement, places d'apprentissage, conditions de travail, etc.) sont des facteurs d'attraction ou de rejet (Held et Maillat, 1984, De Stefanis, 1983).

La structure régionale d'un marché du travail est en effet déterminée par des emplois différenciés selon leur contenu, leur stabilité, le rôle qu'ils jouent dans la carrière des individus et les caractéristiques de la main-d'oeuvre qui les occupe. Dans cette structure, l'allocation des individus aux différents postes se fait selon certaines règles et, dès lors, le fonctionnement du marché de l'emploi dépend de la manière dont cette allocation s'organise. Ces procédures d'allocation, que nous désignons sous le nom de «chaîne de mobilité», donnent lieu à des flux de main-d'oeuvre entre les diverses catégories de postes et d'établissements.

Le concept de chaîne de mobilité indique que ce n'est pas l'emploi en lui-même qui est important pour une région, mais une combinaison d'emplois. L'existence de telles combinaisons permet en effet aux habitants de la région de réaliser leurs ambitions ou apparaît comme attrayante de l'extérieur.

Toutes les entreprises n'offrent pas les mêmes types d'emplois. Certaines sont spécialisées dans le recrutement de jeunes qu'elles forment et auxquels elles ouvrent des perspectives d'avancement, d'autres forment des travailleurs qui les quitteront à la fin de leur apprentissage, certaines n'embauchent que des travailleurs qui disposent d'une expérience professionnelle, plusieurs enfin recrutent des travailleurs non qualifiés quel que soit leur âge, leur passé professionnel, leur formation, etc. Il existe évidemment aussi des entreprises qui offrent plusieurs types d'emplois.

En définitive, une structure locale du marché du travail est déterminée par les entreprises qui sont installées à cet endroit; elles sont des stations dans les chaînes de mobilité (Held et Maillat, 1984, Maillat, 1985).

Il existe donc au niveau régional, des structures du marché du travail différenciées qui expliquent leur plus ou moins grand pouvoir de retenir le capital humain, notamment la main-d'oeuvre disposant du savoir-faire spécifique à la région. L'existence de chaînes de mobilité permet, aux travailleurs qui changent d'établissement ou d'entreprise, de maintenir, de véhiculer et de renouveler le savoir-faire dans la région. Pour cette raison, la mobilité peut s'effectuer à l'intérieur de la région et non pas simplement entre les régions. Quand cette possibilité existe, la région peut conserver dans une large mesure son potentiel de savoir-faire car la main-d'oeuvre en passant d'unétablissement à l'autre est le vecteur de ce savoir-faire. 
Revitalisation des régions de tradition industrielle :

\subsection{L'appareil scientifique régional}

L'analyse des milieux a montré que les comportements innovateurs dépendent de variables définies au niveau local et régional. Dès lors, pour donner vie au processus territorial d'innovation, il faut pouvoir produire et développer les compétencesadéquates. C'estlerôle del'appareil scientifique.Parappareil scientifique territorialisé, il faut entendre la présence, au niveau local, d'un ensemble constitué par d'innombrables et étroites relations entre la science, les activités économiques, l'éducation, la finance et le gouvernement (Stöhr, 1986). Cet ensemble agit comme un support à l'innovation et lui permet de se régénérer sans cesse de manière qu'elle devienne un phénomène continu et auto-entretenu.

L'appareil scientifique apparaît comme particulièrement important pour assurer le renouvellement du savoir-faire.

On comprendra mieux ce rôle si on fait la distinction entre savoir-faire empirique (qui repose sur la relation pratique qu'entretient le travailleur avec l'objet et les moyens de travail) et savoir-faire analytique (qui s'appuie sur un savoir scientifique (Barcet et al., 1985).

Le savoir-faire analytique (intégration de la démarche scientifique dans le processus de travail) est basé sur des connaissances techniques, acquises le plus souvent à travers le système de formation. Un système de formation régional territorialisé, c'est-à-dire adapté à des besoins spécifiques, est donc un élément particulièrement important pour générer et entretenir les compétences nécessaires au processus territorial d'innovation.

Quant aux établissements se consacrant à la recherche, qu'ils soient universitaires, publics ou privés, leur rôle et leur contribution dans le processus régional d'innovation est plus controversé. Souvent citésà l'origine du développement extraordinaire de régions comme la Silicon Valley ou la Route 128, les instituts de recherche semblent pourtant dans bien des cas offrir des avantages plus apparents que réels. Ainsi, en Allemagne, Meyer-Krahmer (1985) a montré que les universités, les écoles polytechniques et les instituts de recherche (publics et privés) ne jouent pas un rôle aussi important en tant que foumisseurs de know-how aux PME, comme on veut bien souvent l'affirmer dans l'opinion. Au Pays-Bas, aucune corrélation significative entre la dispersion de l'innovation et celle des établissements de recherche n'a pu être trouvée. Seules les firmes ayant recours exclusivement à des services de $\mathrm{R} \& \mathrm{D}$ externes ont tendance à obtenir assistance et consultance externes dans leur voisinage (Dieperinck et Nijkamp, 1984). Se basant sur ces considérations, Dieperinck et Nijkamp pensent qu'une politique visant à renforcer le potentiel régional en matière d'innovation basée sur la création d'infrastructures de type scientifique et technique (implantation de centres de recherche, etc...) n'aurait pas d'impact substantiel. Par ailleurs, le développement des nouveaux moyens de communication, en permettant l'accès de plus en plus décentralisé aux informations, devrait ainsi favoriser les régions et les entreprises qui jusqu'à présent n'y avaient pas accès, éliminant en cela les effets de proximité (Planque, 1983). 
Ces considérations, au demeurant fort pertinentes, ne semblent pas valables pour les régions de tradition industrielle. En effet, ces demières, secouées par les mutations technologiques et la divison internationale du travail, ont précisément un besoin accru d'informations scientifiques, techniques et commerciales. Ceci est particulièrement vrai lorsque le tissu régional est composé de PME.

Ces PME, susceptibles de changement technologique, n'intègrent bien souvent pas toutes les fonctions de l'entreprise, en particulier celle de R \& D. Aussi la proximité de centres de recherche peut largement leur favoriser l'accès à cette information. Toutefois, pour que ces effets de proximité jouent un rôle actif, il est nécessaire que l'appareil scientifique local développe des spécialisations en rapport avec les activités locales et que des interfaces soient établis entre les centres de recherche et les entreprises.

\section{Une nouvelle politique régionale}

Au vu de ce qui précède, la revitalisation des régions de traditions industrielles appelle des mesures spécifiques et nouvelles par rapport à la politique traditionnelle visant à attirer des entreprises de l'extérieur. Il s'agit désormais d'agir sur les capacités d'innovation et d'incubation du milieu. Plusieurs voies sont possibles. Signalons celles qui nous paraissent les plus importantes :

\subsection{La stimulation de l'entrepreneuriat local}

L'approche du développement territorial porte une attention toute particulière aux ressources propres à la région. L'entrepreneur et l'esprit d'initiative font partie de celles-ci. Pour être active, une région doit générer des entrepreneurs. Il s'agit donc de promouvoir la volonté d'initiative et d'innovation de la population locale. Plusieurs régions ont ainsi développé des cours d'entrepreneuriat. Certes, on peut estimer que l'esprit d'entreprise ne s'enseigne pas! Toutefois, des enseignements portant sur l'entrepreneuriat peuvent révéler des créateurs d'entreprises et les décider à agir.

Les pépinières d'entreprises ou les centres d'incubation peuvent se révéler également comme particulièrement intéressants pour soutenir de jeunes entreprises, les aider et les conseiller dans les premières phases cruciales de la création d'une entreprise. D'autres formules sont également envisageables comme l'essaimage «spin-off» ou l'intrapreneuriat.

\subsection{Les effets de réseaux}

On sait que les succursales des entreprises extérieures implantées dans une région entretiennent généralement peu de liens avec le milieu régional. Or, les interdépendances entre activités, notamment entre les activités industrielles et les 
activités de services, sont indispensables au bon fonctionnement du processus territorial d'innovation.

Ces phénomènes d'interdépendance doivent être organisés de telle manière qu'il aboutissent au montage d'opérations conjointes entre la finance, la production et le marché.

\subsection{La territorialisation de l'appareil scientifique et l'effet réseau}

L'émergence des technologies nouvelles a des implications pour toutes les régions. C'est une des raisons pour lesquelles de nombreuses régions développent aujourd'hui despolitiques scientifiqueset technologiques adaptéesàleurscompétences età leur savoir-faire. Le but est de mobiliser les institutions de recherche scientifique et d'enseignement supérieur en vue de redynamiser le tissu industriel et tertiaire régional par le transfert technologique et la valorisation des résultats de la recherche. La stimulation de l'innovation au niveau territorial dépend de la capacité du milieu à susciter ou à organiser la collaboration entre l'appareil scientifique, l'appareil de production, les milieux financiers, etc., dans le but d'aboutir à des productions nouvelles et à des créations d'entreprises.

\subsection{Les infrastructures nouvelles}

Les infrastructures permettant de transmettre et de véhiculer l'information sont aujourd'hui indispensables.

On pense notamment aux réseaux télématiques, aux bases de données locales, aux agences de diffusion de l'information technique ou économique. Une région qui ne dispose pas de ces infrastructures qui permettent de capter, de diffuser et de produire l'information, risque de devenir un milieu passif consommant des services conçus et réalisés ailleurs.

En définitive, la politique de revitalisation des régions de tradition industrielle (telles que nous les avons décrites ici) doit viser à reconstituer un système de production complet et cohérent. Complet, parce que comprenant des activités de services et des activités manufacturières articulées de l'amont (recherche et développement) vers l'aval (marché). Cohérent, parce que capable de générer des effets de réseau et de susciter des compétences régionales spécifiques. 


\section{BIBLIOGRAPHIE}

AROCENA, J., BERNOUX, P., MINGUET, C., PAUL-CAVALLIER, M., RICHARD, P., La création d'entreprises, un enjeu local, La documentation française, Notes et études documentaires, no 4709-4710, 1983.

AYDALOT, P., Recherche de nouveaux dynamismes spatiaux, in : AYDALOT P., éd., Crise et espace, Paris, Economica, 1984, pp. 38-59.

AYDALOT, P., Présentation, in : AYDALOT P., Milieux innovateurs en Europe, Paris, GREMI, 1986a.

AYDALOT, P., L'aptitude des milieux locaux à promouvoir l'innovation, in : FEDERWISH, J. et ZOLLER, H., Technologies nouvelles et ruptures régionales, Paris, Economica, 1986b.

BAILLY, A., MAILLAT, D. et REY, M., Tertiaire moteur et développement régional : le cas des petites et moyennes villes, Revue d'Economie Régionale et Urbaine, 1984, no 5, pp. 757-776.

BAILLY, A. et MAILLAT, D., Le secteur tertiaire en question, Genève, ERESA, 1986.

BARCET, A., LEBAS, C. et MERCIER, C., Savoir-faire et changements techniques, Lyon, Presses universitaires de Lyon, 1985.

BOULIANNE, L. et MAILLAT, D., Technologies, entreprises et régions, Saint-Saphorin, Georgi, 1983.

COFFEY, W.J. et POLESE, M., La localisation des activités de bureau et des services aux entreprises : un cadre d' analyse, Revue d'Economie Régionale et Urbaine, 1984, n 5, pp. 717-730.

CORBAT, P., L'incidence de la politique industrielle régionale, EDES, Neuchâtel, 1985.

DECOSTER, E. et TABARIES, M., L'innovation dans un pôle scientifique et technologique, le cas de la cité scientifique Ile-de-France Sud, Aydalot, 1986a.

DESTEFANIS, M., Le fonctionnement du marché de l'emploi au niveau local en France, in : MAILLAT, D., éd., Le fonctionnement du marché de l'emploi au niveau local, Saint-Saphorin, Georgi, 1983.

DIEPERINK, H. et NIJKAMP, P., Spatial dispersion of industrial innovation. A case study for the Netherlands, Communication au colloque de l'ASRDLF, Marrakech, 1985.

DOMMERGUES, P., Le Michigan, laboratoire de l'économie - territoire, Le Monde Diplomatique, 1986, octobre.

GAFFARD, J.-L., Restructuration de l'espace économique et trajectoires technologiques, in : AYDALOT, 1986a.

HELD, D. et MAILLAT, D., Marché de l'emploi, Lausanne, Presses Polytechniques Romandes, 1984.

KEEBLE, D. et WEVER, E., La dynamique de l'insertion des PME innovatrices, Revue d'Economie Régionale et Urbaine, 1986, $\mathrm{n}^{\circ}$ 5, pp. 587-607.

LAMBOY, J.-G., The regional ecology of technical change, communication à la conférence de l'O.N.U., Varsovie, 1984, juin. 
LAMBOY, J.-G. et TORDOIR, P.-P., Professional services and regional development : a conceptual approach, communication à la conférence FASTR-C.E.E., Bruxelles, 1985, octobre.

MAILLAT, D., Les PME innovatrices et la revitalisation des régions, Revue d'Economie Régionale et Urbaine, 1986, $\mathrm{n}^{\circ}$ 5, pp. 688-693.

MAILLAT, D., SCHOEPFER, A. et VOILLAT, F., éd., La nouvelle politique régionale. Le cas de l'arc jurassien, Neuchâtel, EDES, 1984.

MAILLAT, D. et VASSEROT, J.-Y., Les milieux innovateurs, le cas de l'arc jurassien, in : AYDALOT, 1986a.

MEYER-KRAHMER, F., Innovation behaviour and regional indigenous potential, in : Regional Studies, vol. 19, no 6, 1986a, octobre.

OCDE, Rapport analytique sur la recherche, la technologie et la politique régionale, Paris, 1984.

OCDE, Restructuration régionale, Paris, 1986.

PECQUEUR, B., Crise économique : crise du discours sur l'espace, Economie et humanisme, 1986, mai-juin.

PERRIN, J.-C., Un bilan théorique et méthodologique, in : J. Federwish et H.-G. Zoller, Technologies nouvelles et ruptures régionales, Economica, 1986a, pp. 204-215.

PEYRACHE, V., Mutations régionales vers les technologies nouvelles, le cas de la région de Saint-Étienne, in : AYDALOT, 1986a.

PIORE, M.-J. et SABEL, C.-F., The Second Industrial Divide, Basic Book, New York.

PLANQUE, B., (1983), Innovation et développement régional, Paris, Economica, 1983.

PLANQUE, B. ET PY, B., La dynamique de l'insertion des PME innovatrices, Revue d'Economie Régionale et Urbaine, 1986, $\mathrm{n}^{\circ}$ 5, pp. 587-607.

PREEL, B., Pour servir les entreprises que peuvent faire les collectivités locales?, in : Espaces prospectifs, $\mathrm{n}^{\circ} 4,1986$, janvier, pp. 86-116.

QUEVIT, M., Les politiques d'innovation technologique dans les régions de tradition industrielle : le cas de la Wallonie, ronéo, 1985.

RAVEYRE, M.-F. et SAGLIO, J., Les systèmes industriels localisés : éléments pour une analyse sociologique des ensembles de PME, Sociologie du travail, $\mathrm{n}^{\circ} 2,1984$.

REES, J., éd., Technology, Regions and Policy, Rowman et Littlefeld, 1986.

STOHR, W.B., Territorial innovation complexes, in : AYDALOT, 1986a. 